and physical studies, culture, ecology, physiology, biology, morphology, taxonomy and utilization.

\section{The World Health Organization}

"As International Nomenclature of Yaws Lesions", by Dr. C. J. Hackett, in co-operation with an inter. national group of experts and participants of the international conference on yaws control held at Enugu, Nigeria, in 1955 (World Health Organization Monograph Series No. 36. Pp. 103. Geneva: World Health Organization; London: H.M. Stationery Office, 1957. 12 Swiss francs; 20s.; 5 dollars), is an outstanding contribution to the study of the disease called, in English, 'yaws', in French 'pian', in German and Dutch 'frambosia', in Spanish 'buba', and in Portuguese 'bouba'. The various names used in the study of the disease are defined, and the stages in the disease are described. There are seventy-six plates showing the frightful lesions caused by the spirochæte, Treponema pertenue, which is responsible for the disease. Some of these show the remarkable healing without scarring that may follow a single injection of penicillin. The nomenclature supersedes the earlier one given in the World Health Organization Monograph Series No. 5, entitled "Atlas of Frambœia", by K. R. Hill, R. Kodijat and M. Sardadi, published in 1951. The successful reduction of yaws and the campaigns against it sponsored by the Organization and by UNICEF are described in "The First Ten Years of the World Health Organization" (pp. viii $+540+$ 28 pages of photographs. Geneva: World Health Organization; London: H.M. Stationery Office, 1958. 25s.; 5 dollars; 15 Swiss francs), which gives a comprehensive survey of its achievements in the many fields of health control and eradication of disease in which it operates all over the world. Another account of its achievements, illustrated by remarkable photographs of the work actually in progress, and attractively written by Ritchie Calder, is entitled "Ten Steps Forward, World Health 1948-58" (London: H.M. Stationery Office, 1958. 2s. 6d.).

\section{Health in the Tropics}

THE report of the thirty-second meeting of the Ross Institute Industrial Advisory Committee on June 19 (pp. 19. London: London School of Hygiene and Tropical Medicine, incorporating the Ross Institute, 1958) records the address given by $\mathrm{Dr}$. Alan Gilroy, principal of the India Branch of the Institute, and the subsequent discussion. Dr. Gilroy referred to the progress made in regard to malaria. In a large sample of tea-estate labour there were 12 cases of malaria per 1,000 in 1957, no deaths, and a spleen rate of only 1.5 per cent. As a result, with the adoption of a malaria-eradication programme by the Government of India, the industry could now contemplate relief from its recurring expenditure of nearly thirty years. Anæmia remained a most important cause of ill-health, and about 90 per cent of the cases are of simple iron-deficiency anæmia, although the diet was not short of iron and patients were not losing it to hookworm. In replying in the discussion, Dr. Gilroy said that the scientific investigation of malnutrition was as yet incomplete, but it appeared that although the diet was adequate in quantity it was deficient in quality. This was due to ignorance rather than to lack of purchasing power. Dr. Gilroy also said that the stage had been reached when it was accepted and recognized that family planning was necessary, and he also referred to the lecture courses in tropical hygiene for planters and the refresher courses held for tea-estate assistant medical officers.

\section{City and Guilds of London Institute}

THE rapid growth of technical education in Great, Britain and overseas is well brought out in the seventy-eighth annual report of the City and Guilds of London Institute, for the year October 1, 1956, to September 30, 1957 (Pp. $\mathrm{v}+118$. London: City and Guilds of London Institute, Gresham College, 1958). In 1957, 119,948 students took one of the great variety of courses arranged by the Institute compared with 105,815 in 1956 ; it is not surprising to learn that 62.5 per cent of students in Great Britain and Ireland were successful in their examinations, whereas the number of successful overseas students was 48.1 per cent. Of the students, almost half of them $(54,391)$ took courses in electrical subje:ts, 21,715 courses in mechanical engineering, 18,640 in building subjects, and 9,378 in chemistry subjects. Other courses taken were in domestic subjects, printing and food technology. Many of the courses were revised during the year and a number of new ones were added; among the latter is a course for a National Certificate in Agriculture for students who have completed a year at a farm institute. The report also contains details of the insignia awards in technology, the work of the art school and the City and Guilds College, as well as the response to examinations set by the Associated Examining Board for the General Certificate of Education.

\section{The Cosmic Distance Scale}

IN his Warner Lecture before the American Astronomical Society, Dr. Allan Sandage has reviewed the current status of work on the cosmic distance scale (Astrophysical Journal, 127, 513; 1958 ; Sky and Telescope, 17,$275 ; 1958$ ). Hubble's criteria for setting up the distance scale have been re-examined. He used the brightness of the cepheid variable stars in the Andromeda and Triangulum nebulæ, the brightness of individual high-luminosity stars for somewhat more distant galaxies, and integrated apparent magnitudes of still more distant objects. The first criterion was revised in 1952 when Baade showed that classical cepheids were 1.5 magnitudes brighter than had been supposed: this effectively doubled the scale of distances. Other complicating features have since been discovered: the periodluminosity 'curve' seems to be a broad band, and new methods will have to be devised to find out whether individual cepheid variables are under- or overluminous for their periods. One can therefore scarcely rely on the cepheid criterion for the thirty galaxies in which these stars can be seen with the 200-in. telescope.

Sandage has shown that Hubble's second criterion was incorrectly applied, in that many of the objects which Hubble believed to be bright stars were in fact ionized hydrogen regions; their nature only became evident when photographs were obtained through $\mathrm{H}_{\alpha}$ filters. In the nebula $M 100$, for example, objects at magnitude $19 \cdot 0$ are ionized hydrogen regions, not stars, the brightest stars appearing at magnitude $20 \cdot 8$. If these individual stars have absolute magnitudes of $-8 \cdot 5$, the distance of $M 100$ would be 